\title{
Geographien digitaler Alltagskultur. Überlegungen zur Digitalisierung in Schule und Unterricht' ${ }^{1}$
}

\author{
* tilo.felgenhaver@ph-ooe.at, Pädagogische Hochschule Oberösterreich \\ **karsten.gaebler@uni-jena.de, Friedrich-Schiller-Universität Jena, Institut für Geographie ( $\square$ korresp. Autor) \\ eingereicht am: 30.01.2019, akzeptiert am: 28.04.2019
}

Die digitale Revolution ist nicht nur als technologisches, sondern gleichsam als soziales und kulturelles Phänomen zu betrachten. Der Artikel liefert dazu einen Überblick über theoretische Zugänge zu digitaler Kultur und stellt geographische Forschungsarbeiten mit einem soziokulturellen Fokus auf das Digitale vor. Vor diesem Hintergrund werden Schlussfolgerungen für die geographische Bildung in zweierlei Hinsicht diskutiert: Zum einen im Hinblick auf die Digitalisierung der Vermittlungspraxis der Lehrenden. Zum anderen mit Blick auf potentielle neue Unterrichtsthemen.

Keywords: digitale Revolution, Mediengeographie, digitale Kompetenzen

\section{Geographies of digital everyday culture. Thoughts on digitalisation in schools and in lessons}

The so-called "digital revolution" is not only a technological, but at the same time, a social and cultural phenomenon. This article introduces concepts of digital culture and provides an overview on related geographic research from a social science perspective. Against this background, conclusions for current geography education are two-fold: firstly, in the sense of the digitization/digitalization of the practice of teaching. Secondly, the study of the geographies of digital culture may provide new potential topics for the geography curricula.

Keywords: Digital Revolution, Media Geography, digital competencies

Es gehört mittlerweile zum Standardrepertoire sozialwissenschaftlicher Zeitdiagnosen, einen grundlegenden gesellschaftlichen Wandel durch das Aufkommen digitaler Technologien zu verkünden (vgl. z. B. Rifkin 2011; Floridi 2015). Die Verbreitung insbesondere mobiler digitaler Geräte scheint - wenn man den einschlägigen Diagnosen folgt - fast alle Lebensbereiche zu verändern. Wie wir miteinander kommunizieren, soziale Beziehungen gestalten, uns mit politischen Fragen auseinandersetzen, Trennlinien zwischen dem Öffentlichen und dem Privaten ziehen - das gesamte Alltagsleben scheint gegenwärtig einem umfassenden Wandel unterworfen zu sein. Auf den ersten Blick könnte man meinen, die Digitalisierung hätte unsere Art, in der Welt zu sein, fundamental verändert (vgl. Lagerkvist 2017).

Es ist daher kaum überraschend, dass die digitale Wende auch für das Unterrichten und die Ausbildung von Lehrkräften grundlegende Herausforderungen mit sich bringt. Einerseits eröffnen die digitalisierten Lebenswelten neue Möglichkeiten des Lehrens und Lernens. Der Einbezug digitaler Werkzeuge in Unterricht und Schule kann beispielsweise eine stärkere Flexibilisierung und Individualisierung von Lehrangeboten oder eine effizientere Verwaltung und Kommunikation im Schulkontext ermöglichen, geht zugleich jedoch mit neuen Fragen des Datenschutzes und des Informationsmanagements einher. Digitale Alltagskultur, so scheint es, macht an den Schultüren nicht Halt.

Die Durchdringung des (Schul-)Alltags mit digitalen Werkzeugen spiegelt sich aktuell auch in einer

Bei dem vorliegenden Beitrag handelt es sich um eine veränderte und stark erweiterte Fassung der Einführung zum Sammelband "Geographies of Digital Culture" (Felgenhauer \& Gäbler 2018). Wir danken dem Verlag für die Erlaubnis zur Übersetzung von Teilen des Beitrags. 
verstärkten bildungswissenschaftlichen Auseinandersetzung mit den spezifischen Anforderungen für digitales Lehren und Lernen wider. Handreichungen wie beispielsweise das „digi.kompP“ Kompetenzmodell (Brandhofer et al. 2016) thematisieren die Implikationen der Digitalisierung für den Schulalltag und entwickeln Leitlinien zur entsprechenden Ausbildung und Professionalisierung von Lehrkräften. Die dabei skizzierten Kompetenzen digital Lehrender decken erwartungsgemäß einen breiten Bereich ab, von technischem Grundlagenwissen und Anwendungskompetenz digitaler Technologien über didaktische Expertise und sozialwissenschaftliches Reflexionsvermögen bis hin zu Organisations- bzw. Verwaltungskompetenzen.

Parallel zu dieser umfassenden Digitalisierung der Vermittlungspraxis und ihrer didaktischen Reflexion wird im Schulkontext zunehmend jedoch auch die Frage gestellt, inwiefern der digitale „Turn“ nicht nur als neuer Modus des Lehrens und Lernens aufzufassen ist, sondern auch als Gegenstand und Inhalt des Unterrichts. Besonders für die Geographie und den GWUnterricht, so möchten wir in diesem Aufsatz argumentieren, eröffnen sich durch eine Thematisierung der digitalen Wende und der mit ihr verbundenen digitalen Alltagskultur neue Perspektiven. So etwa wird in der fachwissenschaftlichen Debatte schon seit längerem darauf hingewiesen, dass die Integration digitaler Technologien in den Alltag eng mit neuen Raumverhältnissen verbunden ist, d.h. mit neuen Wahrnehmungen räumlicher Konfigurationen, neuen räumlichen (oder körperlichen) Praktiken oder mit neuen geographischen Verbreitungsmustern digitaler Infrastrukturen. Diese räumliche Dimension des Digitalen knüpft dabei einerseits an etablierte geographische Problemstellungen an, wirft zugleich jedoch neue Fragen auf, zu deren Bearbeitung unserer Auffassung nach weniger eine technikorientierte denn eine sozial- und kulturwissenschaftlich fundierte Perspektive notwendig ist.

Eine sozial- und kulturwissenschaftliche Aufweitung geographischer Fragestellungen im Unterricht setzt das Digitale nicht als selbsterklärend voraus, sondern unterzieht Grundbegriffe, zeitdiagnostische Vorannahmen und disziplinäre Verortungen der digitalen Wende einer kritischen Prüfung. An welche gesellschaftlichen und raumbezogenen Prozesse knüpft digitale Alltagskultur an? Worin unterscheidet sie sich von (vermeintlich) prä-digitalen Lebenswelten? Welche Rolle spielen Raum und das Materielle in der digitalen Alltagskultur? Und schließlich: Wie lässt sich das Digitale geographisch thematisieren? In welchen Teilbereichen der Geographie wird es behandelt? Welche Raumverständnisse kommen dabei zum Tragen?

Wir wollen im Folgenden einen solchen Zugang zu den Geographien digitaler Alltagskultur skizzieren und einen Überblick über aktuelle fachwissenschaftliche Arbeiten geben. Dazu gilt es in einem ersten Schritt, das Digitale nicht primär als technisches, sondern als sozial-kulturelles und historisches Phänomen zu deuten. Hierzu versuchen wir neben zeitdiagnostischen Charakterisierungen des Digitalen Schlüsselmerkmale und Grundbegriffe der digitalen Alltagskultur herauszuarbeiten. Im zweiten Schritt sollen auf dieser Grundlage geographische Zugänge zum Digitalen systematisiert und mögliche Themeninhalte - eben im Sinne von sozial-kulturellen Geographien des Digitalen - für den GW Unterricht erschlossen werden. Abschließend erweitern wir die an geographischen Fragen entwickelte Perspektive und zeigen am Beispiel des „digi.kompP“-Kompetenzmodells, wie ein sozial- und kulturwissenschaftlich fundierter Umgang mit Schule als digitalisierter Lebenswelt allgemein aussehen kann.

\section{Digitale Alltagskultur in kritischer Perspektive}

Die Selbstverständlichkeit der gegenwärtig so populären Rede von einer „digitalen Revolution“ kann weder aus geographischer noch aus sozial- und kulturwissenschaftlicher Sicht befriedigen. Zweifelsohne hat die Digitalisierung in vielen Feldern des Alltags bislang ungekannte Möglichkeiten eröffnet, sodass sich inzwischen zu Recht von einer digitalen Alltagskultur sprechen lässt. Der Einsatz digitaler Technologien hat sich z. B. zu einem grundlegenden Mittel der sozialen Integration entwickelt. Um ein als „normal“ angesehenes Leben zu führen, bedarf es in den meisten Kontexten des Besitzes digitaler Geräte und eines möglichst schnellen Internetzugangs. Und in geographischer Hinsicht scheinen digitale Technologien alltägliche Phänomene zunehmend zu enträumlichen. So bieten virtuelle öffentliche Räume neue politische Ausdrucksformen und erweitern damit das Spektrum der politischen Partizipation und des Protestes (siehe z. B. Maireder \& Ausserhofer 2014; Lee et al. 2017; Kavada 2016). Digitalisierte Kommunikation ermöglicht indessen die gleichzeitige Integration in räumlich voneinander entfernte Gemeinschaften und fördert so multi-lokale Lebensstile bis hin zur Aufrechterhaltung von Intimoder Familienbeziehungen über Distanz (vgl. Beck \& Beck-Gernsheim 2011). Die Digitalisierung der Arbeit („Arbeit 4.0“) hingegen verwischt zunehmend die räumlichen Grenzen von Arbeits- und Privatsphäre und erzeugt neue Freiheiten wie auch neue Formen der Ausbeutung (vgl. z. B. Brynjolfsson \& McAfee 2011, 2014; Dyer-Witheford 2015; Huws 2003).

Allerdings: Die digitalisierten Alltagswelten unserer Gegenwart sind keineswegs enträumlicht, wie es 
manche Zeitdiagnose vorschnell behauptet, sondern digitale Technik und ihre Nutzung erzeugen eigene räumliche Konfigurationen und reproduzieren zum Teil ganz „undigitale“ Raumverhältnisse. So zum Beispiel beruht der Zugang zum Internet auf ungleich verteilten materiellen Infrastrukturen (man denke etwa an die berüchtigte „digitale Kluft" zwischen ländlichen und urbanen Räumen). Durch Praktiken der Geocodierung oder des Geoblockings hingegen werden territoriale Grenzen im Internet eingeführt und damit ganz klassische, ,analoge“ Kontrollstrategien angewendet. Allein diese Beispiele deuten an, dass die Rede von einer digitalen Wende alles andere als unproblematisch ist. Statt einer alles erfassenden Revolution vermischen sich gegenwärtig digitale Praktiken mit „vordigitalen“ Formen sozialer Organisation, Strategien der Überwachung und Regulierung, Mustern sozialer Schichtung usw.

Dass die Idee einer digitalen Revolution unterkomplex und historisch kurzsichtig bleibt, zeigt auch ein Blick in technikgeschichtliche Arbeiten (vgl. z. B. Rid 2016; Park et al. 2011). So etwa stellt die mit gegenwärtigen Technologien ermöglichte globale „Echtzeitkommunikation" natürlich keine fundamental neue geographische Erfahrung dar, sondern setzt die Raumlogik bereits zur Mitte des 19. Jahrhunderts etablierter Technologien fort (vgl. z. B. Marvin 1988). Beispiele wie die Entwicklung des weltumspannenden Telegraphennetzes im 19. Jahrhundert zeigen, wie problematisch die mit der Rede "disruptiver" Veränderungen suggerierte Unterscheidung einer digitalen Gegenwart von einer analogen Vergangenheit ist - und sie machen auf die Notwendigkeit einer Historisierung gegenwärtiger digitaler Praktiken aufmerksam.

Eine solche Kontextualisierung des Digitalen erscheint dabei nicht nur mit Blick auf Praktiken und Infrastrukturen sinnvoll, sondern auch in wissensbzw. wissenschaftsgeschichtlicher Hinsicht. Auch hier lässt sich bereits bei kursorischer Lektüre festhalten, dass Fragen nach der Verbreitung, Nutzung und den Konsequenzen digitaler Technologien in den Sozialund Kulturwissenschaften nicht erst mit der „Digitalen Revolution“ am Beginn des 21. Jahrhunderts aufgekommen sind. So wurden bereits seit Mitte der 1970er Jahre im Zuge der zunehmenden Verbreitung von Personalcomputern oder seit den 1990er Jahren mit der Entwicklung des World Wide Web Fragen des organisatorischen Wandels aufgeworfen (vgl. z. B. National Academy of Engineering 1983; Morell 1988; Berghel 1997), oder aber es wurde die wachsende Bedeutung von Information für moderne Gesellschaften thematisiert (vgl. etwa Manuel Castells' Trilogie „Das Informationszeitalter“, insbesondere Castells 2001). Seit Mitte der 2000er Jahre konzentriert sich ein wachsender Teil der sozial- und kulturwissenschaftli- chen Literatur zu digitalen Technologien dann auf digitale Alltagspraktiken und das Aufkommen einer digitalen Alltagskultur (vgl. z. B. Poster 2006; Couldry 2012: 33 ff.; Horst \& Miller 2013). Daneben ist in jüngerer Zeit ein verstärktes theoretisches Interesse an den Beziehungen von Technologie und Gesellschaft und den Prozessen sozio-technischer Ko-Evolution zu verzeichnen (vgl. z. B. Miller 2011; Berry 2013; Dolata 2013; Matthewman 2011).

\section{Schlüsselmerkmale digitaler Alltagskultur}

Eine an den genannten Orten dabei immer wieder thematisierte Schlüsselfrage zum Verständnis der gegenwärtigen Digitalisierungsprozesse und ihrer gesellschaftlichen Bedingungen und Konsequenzen ist, worin die Eigenart digitaler Techniken bzw. der digitalen Alltagskultur besteht. Aus der Vielzahl der in der Literatur diskutierten Aspekte (vgl. z. B. Miller 2011) erscheinen aus geographischer Sicht insbesondere die folgenden Elemente bedeutsam: (1) Digitalität, (2) Netzwerkcharakter und (3) Mobilität.

\subsection{Erstes Schlüsselmerkmal: Digitalität}

Es ist natürlich kaum überraschend, dass Digitalität und Digitalisierung zentrale Merkmale der Alltagswelt nach dem Digital Turn sind. Es ist jedoch bemerkenswert, wie unbestimmt der Begriff des Digitalen in der öffentlichen wie wissenschaftlichen Debatte häufig bleibt. „Digitalisierung“ steht beispielsweise für die Verbreitung von Computersystemen und PCs in der öffentlichen Verwaltung seit den 1970er Jahren, aber gleichermaßen auch für die zu Beginn des 21. Jahrhunderts einsetzende Durchdringung alltäglicher Lebenswelten mit vernetzten digitalen Geräten sowie deren soziale, kulturelle oder wirtschaftliche Auswirkungen (Passig \& Scholz 2015). Diese Verwendungsweise des Begriffs der Digitalisierung geht primär von der Sicht der Nutzer/innen aus und beschreibt technologischen Wandel als Oberflächenphänomen, ohne dabei auf die zugrundeliegenden technischen Verfahren einzugehen.

Eine stärker technisch orientierte Verwendungsweise des Begriffs findet sich da, wo von „Digitalisierung“ als Umwandlung von analogen Daten in ein binäres numerisches System - beispielsweise von Einsen und Nullen - die Rede ist. Diese Vorstellung von Digitalisierung geht auf das lateinische Substantiv „digitus“ (Finger) zurück und verweist damit auf den Akt des Zählens mit der Hand. In der englischen Sprache wird der Prozess der Transformation von Informationen in binäre Werte daher oft als „digitization“ bezeichnet, 
im Gegensatz zur „digitalization“, die die sozialen, kulturellen oder wirtschaftlichen Folgen der Digitalisierung bezeichnet (Brennen \& Kreiss 2016).

Die Digitalisierung als Übersetzung von kontinuierlichen in diskrete numerische Werte stellt zunächst einmal einen Prozess der Reduktion von Komplexität dar. Digitale Informationsverarbeitung lässt sich rein technisch also mit jeder Vorrichtung ermöglichen, die zwei unterschiedliche Zustände darstellen kann. Nimmt man den Begriff ernst, liegt digitale Kommunikation also auch unabhängig der Nutzung und Verfügbarkeit von elektrischer Energie vor - beispielsweise bei optischen Telegraphieanlagen wie dem Heliographen oder der Flaggentelegraphie (vgl. Maddalena \& Packer 2015: 94 ff.). Was mit diesen Technologien erstmals möglich wird, ist die Trennung der Informationsübertragung von der Mobilität eines physischen Informationsträgers. Digitalität ermöglicht damit die schnelle Kommunikation über große Entfernungen und wird so zum geographisch relevanten Phänomen.

Eine weitere folgenreiche Eigenschaft der Digitalisierung von Daten ist die durch sie beförderte Quantifizierung und Berechenbarkeit („Computation“). Viele der Merkmale, die gegenwärtig mit dem Digitalen assoziiert werden, scheinen eher ein Effekt von Quantifizierung und Berechnung als der bloßen Digitalität (im oben genannten Sinne) zu sein. Die automatisierte Datenverarbeitung etwa - von einfachen Algorithmen bis hin zu den berüchtigten „Big Data“-Anwendungen - prägt heute einen großen Teil unserer digitalen Alltagserfahrungen (vgl. z. B. Amoore \& Piotukh 2016; Seyfert \& Roberge 2017). Was uns von digitalen Medien als relevante Information präsentiert wird, welche Nachrichten und Anzeigen wir lesen, welche Suchergebnisse wir erhalten etc. ist wesentlich das Ergebnis algorithmisch analysierter (Nutzungs-) Daten. Wie insbesondere von Digitalisierungskritikern und -innen (vgl. z. B. O’Neil 2016) hervorgehoben wird, besitzen Algorithmen dabei eine zunehmende Wirkmächtigkeit und erlangen scheinbar eigenständige Handlungsmacht. Auch hier gilt allerdings: Algorithmen sind nicht per se an elektronische Technologien gebunden, sondern ihre Grundidee - die Festlegung von Handlungsschritten zur Lösung eines bestimmten Problems - ist wesentlich älter. Ihre technische Realisierung lässt sich mindestens bis zu den mechanischen Rechenmaschinen des 18. Jahrhunderts zurückverfolgen. Als prominentestes Beispiel sei hier an die in der ersten Hälfte des 19. Jahrhunderts entwickelte "Analytical Engine“ von Charles Babbage erinnert, die die heutige Idee eines Computers als automatische Rechenmaschine bereits rein mechanisch verwirklichte (Burckhardt 2017: $50 \mathrm{ff}$.).

Es lässt sich also bereits mit wenigen technikhistorischen Hinweisen zeigen, dass die Gegenüberstellung von analog und digital, mit der die Gegenwart häufig von einer Vergangenheit vor der digitalen Wende abgegrenzt wird, problematisch ist. Was analog und was digital ist, hängt vielmehr von der Perspektive ab, die gewählt wird.

\subsection{Zweites Schlüsselmerkmal: Netzwerkcharakter}

Das zweite Schlüsselelement digitaler Alltagskultur ist die Vernetzung. Die Integration digitaler Geräte in den Alltag hat spätestens seit Beginn der 1990er Jahre mit dem öffentlichen Zugang zu globalen Kommunikationsnetzen wie dem Internet erheblich zugenommen (vgl. etwa Kohut et al. 1995 für das Beispiel der USA). Ohne die Vernetzung von Akteuren und Akteurinnen dürfte die digitale Alltagskultur kaum mehr denkbar sein - und inzwischen werden sogar nichtmenschliche Akteur/innen in ein „Internet der Dinge" integriert.

Es braucht jedoch auch mit Blick auf die Vernetzung wenig Phantasie um zu sehen, dass konnektive Praktiken und das Schaffen von Netzwerken kein Privileg des 21. Jahrhunderts sind. Ganz im Gegenteil: Begreift man unter dem Begriff der Vernetzung zunächst einmal die Herstellung von Verbindungen zwischen zwei oder mehr Entitäten und die Etablierung von Zirkulationsprozessen zwischen diesen Punkten, dann muss Vernetzung als eine grundlegende, fast unvermeidliche menschliche Praxis aufgefasst werden. Und selbst wenn man die Idee des Netzwerks in einem engeren Sinne als Charakteristikum digitalisierter Lebenswelten versteht, weist sie historisch weit über das 21. Jahrhundert hinaus. Sebastian Giessmann beispielsweise macht darauf aufmerksam, dass das „Netzwerk“ als Interpretationsmodell spätestens seit dem 18. Jahrhundert Verwendung findet (Giessmann 2014: 17; vgl. auch Barkhoff et al. 2004). Der Historiker Jürgen Osterhammel (2009: 1010 ff.) hingegen verbindet das Konzept des Netzwerks eng mit dem "Wandel der Welt" im 19. Jahrhundert, insbesondere mit der Entwicklung globaler Zirkulationssysteme in den Bereichen Verkehr und Kommunikation (z. B. Dampfschifffahrt, Eisenbahn, Telegraphie), Handel (z.B. stabile internationale Handelsbeziehungen) sowie Geld und Finanzen (z. B. internationale Währungssysteme). Netzwerke seien demnach wesentlich mit Globalisierungsprozessen verbunden und seien Produkte der Moderne.

Was die gegenwärtigen digitalen Vernetzungspraktiken von ihren historischen Vorläufern unterscheidet, ist bestenfalls ihre Geschwindigkeit bzw. ihr Umfang. Digitale Kommunikation ist heute z. B. oft bidirektional und interaktiv, d.h. Inhalte können mit einer Vielzahl von Beteiligten ausgetauscht und Informationen können mehr oder weniger mühelos geteilt wer- 
den. Die für den Einzelnen/die Einzelne potenziell verfügbaren Informationen werden in digitalen Netzwerken multipliziert und die Informationsmonopole der traditionellen, unidirektionalen Medien werden damit zunehmend in Frage gestellt. Dass sich damit jedoch nicht automatisch utopische Visionen einer Demokratisierung durch digitale Vernetzung verwirklichen, darauf machen in jüngerer Zeit insbesondere die Debatten um Internetüberwachung oder sogenannte „Fake News" aufmerksam.

Die Vernetzung von Akteuren und Akteurinnen erlangt mit den neuen digitalen Technologien schließlich noch in einem weiteren Sinne geographische Relevanz: Der technisch vermittelte Austausch von Information kann zunehmend als Ko-Präsenz trotz räumlicher Distanz erlebt werden. Insbesondere die mit Breitband-Internetverbindungen beförderte Kombination akustischer und visueller Kommunikationskanäle ermöglicht eine Telepräsenz (Minsky 1980), d. h. die scheinbare Anwesenheit über Distanz und damit die vermeintliche Irrelevanz des physischen Standorts (vgl. Miller 2011:31 f.). Doch auch hier gilt es zu bemerken, dass die Vermischung von Onlineund Offline-Praktiken im Alltag räumliche Bezüge nicht aufhebt, sondern eher die Grenze zwischen physischer und virtueller Präsenz verwischt und so ganz eigene Geographien erzeugt.

\subsection{Drittes Schlüsselmerkmal: Mobilität}

Das dritte Schlüsselmerkmal gegenwärtiger digitaler Technologien ist Mobilität. Mit der fortschreitenden Miniaturisierung von Rechentechnik und dem Aufkommen des drahtlosen Internetzugangs - idealtypisch repräsentiert durch das Smartphone - verbreiteten sich neue Alltagspraktiken wie etwa das Textmessaging, die Nutzung von „Augmented Reality"-Anwendungen oder die Satellitennavigation. „Wireless" scheint zu einem Markenzeichen der neuen, digitalen Alltagskultur geworden zu sein.

Drei Aspekte sind bei dieser Mobilisierung aus geographischer Sicht besonders hervorzuheben. Der erste bezieht sich auf das materielle Rückgrat der scheinbar immateriellen mobilen Medien. Obwohl das Internet im Alltag oft mit Virtualität assoziiert wird - man denke nur an die Symbolik der schwerelosen „Cloud“ - und Drahtloszugang zum Netz häufig den Eindruck erweckt, das Internet sei „einfach so da“, ist letztlich nur der kleinste Teil der technischen Infrastruktur des mobilen Internetzugangs transportabel. Die globale Internetkommunikation basiert beispielsweise auf einem, der Telegraphieinfrastruktur des 19. und frühen 20. Jahrhunderts sehr ähnlichen, Unterseekabelnetz (vgl. Starosielski 2015a) sowie auf einer unzähligen Masse an Funkmasten, Übertragungsstationen usw.
Auch das drahtlose Internet zu Hause, das sich so scheinbar mühelos und bar fast jeder Materialität nutzen lässt, setzt ein bis zum eigenen Hausanschluss ausgebautes Breitbandkabelnetz voraus (was in der Literatur häufig als „Last mile problem“ diskutiert wird). Was zunächst also mobil, schwerelos und immateriell erscheint, ruht auf einer letztlich erstaunlich stationären bzw. trägen materiellen Basis.

Es darf daher nicht überraschen, dass das im Alltag oft unsichtbare materielle Rückgrat mobiler digitaler Technologien untrennbar mit Fragen raumbezogener Gerechtigkeit verbunden ist (vgl. Soja 2010; Alizadeh et al. 2014). Die ungleiche Verfügbarkeit von Breitbandinternetzugängen beispielsweise macht auf den fragilen Charakter der - vom Marketing häufig als Normalfall verkauften - digitalen Alltagskultur aufmerksam. Wer ohne schnelles Internet bleibt, wird im wahrsten Sinne des Wortes abgehängt. Die materielle Dimension digitaler Technik und die mit ihr verbundene Immobilität und Trägheit darf daher nicht unberücksichtigt bleiben, da mit ihr zentrale Fragen nach Gerechtigkeit und Teilhabe aufgeworfen werden.

Ein zweiter mit der Mobilität des Digitalen verbundener Aspekt ist die zunehmende Verschmelzung von Subjekten und Objekten (oder Personen und Dingen) in der alltäglichen Nutzung digitaler Technik. Mobile, meist am Körper getragene Endgeräte werden beispielsweise zunehmend dazu genutzt personalisierte Daten zu erfassen, zu speichern und zu übertragen. Dabei muss es sich nicht einmal um das von der Technikkritik oft befürchtete Ausspähen der Nutzer/innen handeln, sondern auch - und wahrscheinlich in der Mehrzahl der Fälle - wird dies durch freiwilliges „SelfTracking" ermöglicht (vgl. Neff \& Nafus 2016; Lupton 2016). Smartphones und ähnliche Geräte werden als Hüter persönlicher, oft intimer Informationen gegenwärtig nicht nur zu einem Teil der Identität von Subjekten, sondern sie dienen auch als Erweiterung des Körpers. Mit der Nutzung mobiler Geräte geht dabei häufig nicht nur eine Veränderung körperlicher Praktiken einher (vgl. etwa Byington \& Schwebel 2012 zum Fußgänger/innenverhalten), sondern auch eine Veränderung des Körpers selbst (vgl. Hansraj 2014 zu Wirbelsäulenschäden durch die Nutzung von Smartphones).

Die Potenziale mobil nutzbarer digitaler Endgeräte sind dabei durchaus ambivalent zu bewerten. Einerseits ermöglichen individuell (und häufig automatisch) gesammelte Geodaten neue Formen des kollektiven Kartierens und die Bereitstellung einer Vielzahl raumbezogener Informationen. Andererseits erzeugt diese sogenannte „volunteered geographic information" (VGI) auch neue Fragen des Datenschutzes und der Überwachung (vgl. z. B. Blatt 2015). 
Ein dritter und letzter Aspekt der Mobilität des Digitalen ist schließlich die durch sie besonders hervorgehobene Bedeutung des Kontexts alltäglicher Praktiken. Da die Nutzung digitaler Technik nicht mehr in festen - und meist eben auch bekannten - Kontexten stattfindet (wie bspw. im Falle des Festnetztelefons), müssen bei mobiler Kommunikation Kontextinformationen explizit gemacht werden. Mobiltelefonanrufe beginnen daher häufig mit der Frage „Wo bist du?", um einen gemeinsamen Gesprächskontext zu erzeugen oder zu simulieren (Laurier 2001: 494). Es scheint in sozialen Interaktionen jedenfalls von besonderem Interesse zu sein, den Ort des Interaktionspartners oder der Interaktionspartnerin zu kennen. Auch hier lässt sich festhalten, dass die Nutzung digitaler Technologien räumliche Bezüge nicht außer Kraft setzt, sondern neue Geographien hervorbringt.

Die hier nur kursorisch dargestellte (neue) Geographizität des Digitalen spiegelt sich schließlich auch in der Geographie und ihren Teildisziplinen wider. Die in den verschiedenen Teilbereichen der Disziplin entwickelten Ansätze unterscheiden sich dabei z.T. erheblich in Bezug auf ihre Vorstellungen von Raum und Ort, die Rolle von Materialität und Subjektivität sowie ihre politischen Ansprüche oder ihr kritisches Potenzial.

\section{Aktuelle Forschungen zu den Geographien des Digitalen}

Der gegenwärtige Stand geographischer Forschung über das Digitale dokumentiert sich in unterschiedlichen Subdisziplinen, so zum Beispiel in der Kulturund Sozialgeographie, in der Geoinformatik und auch in der Tourismus- und Mediengeographie. Die Arbeiten sind teilweise theoretisch-konzeptionell angelegt, überwiegend jedoch empirisch-technischer Art. Letzteres ist schon aufgrund der Fachtradition der Geographie als in hohem Maße empirisch-technisch geprägter Wissenschaft wenig überraschend. Ebenso wie analoge Technik die empirische Forschung der Geographie geprägt hat, formen digitale Techniken und Medien aktuelle empirische Forschungszugänge. Neu bzw. relativ jung ist aber der Diskurs um die digitale Konstruktion des Räumlichen in Alltag und Gesellschaft, der durch die sozial- und kulturwissenschaftlichen Teildisziplinen der Geographie zunehmend begleitet wird. Digitale Technik wandelt sich dabei vom Instrument zum Gegenstand der Forschung. Die raumtransformierende und letztlich raumerzeugende Kraft des Digitalen wird beispielsweise von Martin Dodge und Rob Kitchin (2005: 162) mit dem Terminus der "transductive practices“ und von Agnieszka Leszczinsky (2015) mit ihrem Konzept einer „spatial media/tion" adressiert. Diese Begriffe betonen, dass während des Handelns mit digitaler Technik und digitalen Medien nicht nur neue Bezugnahmen auf Raum entstehen, sondern diese Praktiken letztlich neue Räumlichkeiten erzeugen. Dieser transformative Charakter digitalisierter Alltagspraxis wird aktuell beispielsweise mit Bezug auf die Bereiche Mobilität, Infrastrukturen, Konsum, Ortswahrnehmung und Images, und Politik und Partizipation untersucht.

Um in diesem Feld eine überblicksartige (und vorläufige) Orientierung zu ermöglichen, werden hier Arbeiten zur (1) Theorie und Begriffsarbeit, (2) zur räumlichen Verteilung digitaler materieller Infrastrukturen als gleichermaßen Ergebnis und Bedingung digitaler Alltagskultur, (3) zu subjektiven Wahrnehmungen und Aneignungen des Räumlichen sowie (4) zur sozialen und kulturellen (damit intersubjektiven bzw. überindividuellen) Konstruktion von digitaler Räumlichkeit aufgeführt. Ungeachtet der Überschneidungen zwischen diesen Kategorien lassen sich mit ihrer Hilfe zahlreiche Einzelthemen zusammenfassen und Unterschiede in den Forschungslogiken und -interessen deutlich erkennen.

\subsection{Theoretisch-konzeptionelle Beiträge zur Geographie des Digitalen}

Im Bereich der theoretischen und konzeptionellen Arbeiten haben zwei Arbeiten in den letzten Jahren besondere Aufmerksamkeit erlangt: Paul C. Adams (2009, 2010) hat mit seiner Propagierung eines „communicational turn“ für die Kultur- und Sozialwissenschaften im Allgemeinen und die Geographie im Besonderen eine Neuausrichtung angestoßen. Dabei geht es um ein Wechselverhältnis zwischen Medien und Welt, aus dem er für die Geographie neue Unterscheidungsmöglichkeiten entwickelt: „communications fill the world and the world fills communications" (Adams 2009: 214). Daraus ergeben sich mit der Verschränkung der in der internationalen Geographie gebräuchlichen Unterscheidung zwischen "place“ (angeeigneter und mit sozialer Bedeutung aufgeladener Raum) und „space“ (abstrakter, objektivierter und vermessbarer Raum) vier Kategorien für die Geographie der Medien (siehe Abb. 1): (a) die Repräsentation von Orten in den Medieninhalten („places in media“; z. B. Images von Orten in der Tourismuswerbung), (b) die Aufladung und Ausstattung „realräumlicher" Orte mit digitalen Medien und Inhalten („media in places"; z.B. CCTV-Überwachung des öffentlichen Raumes), (c) die Verteilung materieller, medienrelevanter Infrastrukturen im Raum („media in space“; z.B. Backbone-Netzwerke) sowie (d) die Einführung räumlicher Abstraktionen in medialen Umwelten („spaces in media“; z. B. Cyberspace oder virtueller 
Raum). Der Nutzen dieses Schemas liegt dabei nicht nur in der Möglichkeit, Forschungsfelder und Themen in logisch unterschiedene Schubladen sortieren zu können, sondern zeigt sich vor allem dann, wenn es gilt, verschiedene Aspekte eines konkreten empirischen Gegenstandes aufzuschlüsseln.

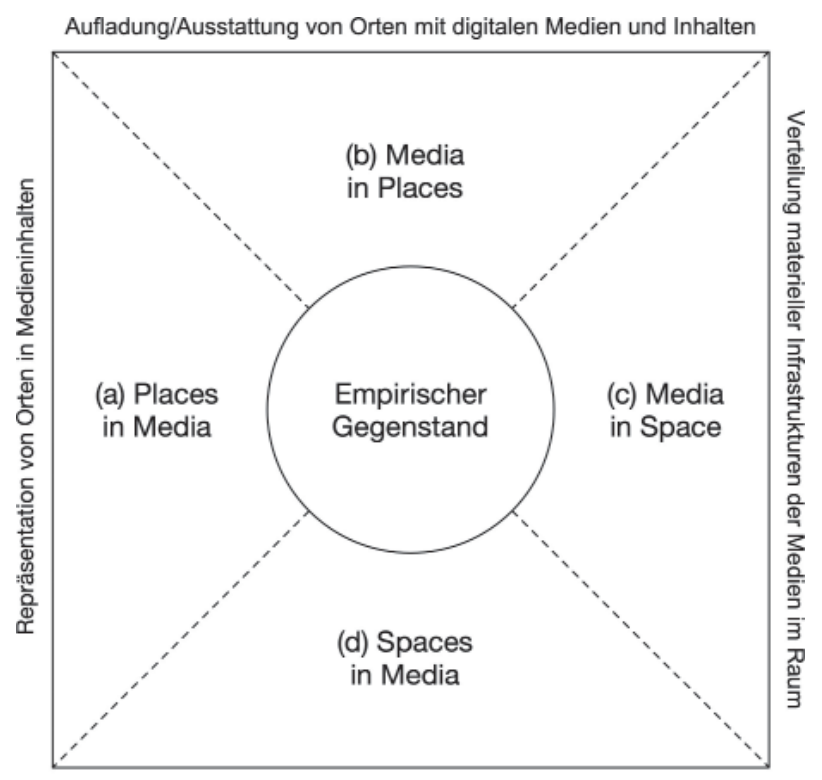

Räumliche Abstraktionen in medialen Umwelten

Abb. 1: Mediengeographische Zugänge (eigener Entwurf auf der Grundlage von Adams 2009: 40)

Ein weiteres übergreifendes theoretisches Schema hat sich aus der aktuellen Neuinterpretation des von Adams diskutierten medialen Turns als digitalem Turn ergeben (Ash et al. 2016). Damit ist die Forschung zu „digitalen Geographien“ als neues Programm ausgegeben, indem nunmehr die Verschränkungen des Digitalen mit dem Räumlichen reflektiert und theoretisch aufgeschlüsselt werden. James Ash et al. (2016) schlagen dies entlang dreier Kategorien vor: Zunächst in Form der Untersuchung von Geographien, die durch die Einführung des Digitalen in geographische Forschungsmethoden und Werkzeuge entstehen (,geographies through the digital“; ebd.: $3 \mathrm{ff}$.). So sind seit der so genannten quantitativen Revolution in der Geographie Ende der 1960er Jahre zahlreiche Schritte hin zu einer Mathematisierung, Formalisierung und rechnergestützten Modellierung als Teil wissenschaftlich-geographischen Arbeitens vollzogen worden. Geographische Informationssysteme (GIS) und aktuelle Forschungsmöglichkeiten um „Big Data" schreiben diese Entwicklung fort. Ash et al. betonen, dass diese Veränderungen nicht nur rein als Weiterentwicklung technischer Möglichkeiten zu beleuchten, sondern mit Blick auf ihren sozialen Kontext auch kritisch zu reflektieren sind. Dazu hat beispielsweise die fachtheoretische internationale De- batte um die Implikationen der quantitativen Revolution und die Entwicklung von „critical GIS“ beigetragen. Geographien die vom Digitalen produziert werden („geographies produced by the digital"), bilden die zweite Kategorie (Ash et al. 2016.: 5 ff.). Damit sind räumliche Verhältnisse gemeint, die sich konkret materiell ausprägen, wie etwa die digitale Erreichbarkeit von Orten, die Erzeugung von Peripherien und räumlichen Ungleichheiten der Versorgung mit digitaler Infrastruktur. Die Diskussionen um vielfältige Formen von „digital divides“ entsprechen beispielhaft dieser Kategorie. Geographien des Digitalen in ihrer dritten Form "geographies of the digital“ (ebd.: $8 \mathrm{ff}$.) meinen nun im engeren Sinn die medialen und subjektiven Geographien des Alltags, die sich aus den Routinen des Gebrauchs digitaler Technologien und Medien ergeben. Hier geht es um die lebensweltliche Aneignung, Wahrnehmung und subjektive Herstellung bzw. Verknüpfung von Orten und Räumen - sowohl real als auch virtuell. Das Digitale ist demnach also weder Mittel des wissenschaftlich-geographischen Zugriffs auf die räumliche Wirklichkeit („geographies through the digital"; ebd.: $3 \mathrm{ff}$.) noch materieller Teil einer transformierten Landschaft räumlicher Verteilungen und Ungleichheiten (,geographies produced by the digital"; ebd.: 5 ff.), sondern Element der Konstruktion des Räumlichen - integriert in zahllose Alltagspraktiken von Laien und deren Lebensstile.

Der Nutzen von theoretischen Unterscheidungen wie sie von Paul Adams oder James Ash et al. geliefert werden kann in einer gesteigerten Aufmerksamkeit für bisher vernachlässigte Analysedimensionen bestehen. Zudem können Brücken und Brüche zwischen den Kategorien expliziter herausgearbeitet werden: zwischen dem Materiellen und Symbolischen, zwischen Kontext und Content des Handelns, oder auch zwischen dem Digitalen als Mittel oder Gegenstand der Forschung. In diesem Sinne können die analytischen Kategorisierungen als verschiedene Wege verstanden werden, auf denen Geographien digitaler Alltagskultur konstruiert werden. Beispielsweise sind räumliche Imaginationen von Orten und Räumen Teil digitalisierter Medieninhalte, ebenso wie Räume und Orte umgekehrt materielle Objekte „enthalten“, die das Digitale in seiner Funktion und Präsenz erst ermöglichen. Die skizzierten analytischen Kategorien sind in ihren Verschränkungen zu untersuchen, um das komplexe Phänomen der Geographien digitaler Kultur verstehen zu können.

\subsection{Studien zur räumlichen Verteilung digitaler Infrastrukturen}

Forschung zur räumlichen Verteilung materieller Infrastrukturen erscheint als ein konzeptionell klar ab- 
grenzbares - und im Wortsinne ausgedehntes - empirisches Forschungsfeld. Klassische geographische Zugangsweisen erfassen die beobachtbaren Objekte und Spuren, die für umfassendere kulturelle, ökonomische und technische Transformationen im Raum bzw. auf der Erdoberfläche stehen. Als ein Beispiel, welches dieser Logik entspricht, kann der aus der (vermeintlich) „vor-digitalen“ ̈̈ra stammende Begriff des "machine space“ (nach Ronald Horvath 1973) gelten, mit dem der Autor plastisch in Text und Karte die Dominanz des Automobils im US-amerikanischen Stadtraum vor Augen führt. Indem Horvath die Räume für den PKW-Verkehr, für Parken, Wartung und Versorgung genau ausweist und kartiert, zeigt er eindrucksvoll die kritischen Aspekte einer solch einseitigen Nutzung und Gestaltung des Stadtraums. Raumdominanz, so der Subtext der Arbeit, bedeutet letztlich gesellschaftliche und wirtschaftliche Dominanz - mindestens im urbanen Kontext mit der dort spürbaren Konkurrenz zwischen verschiedenen Nutzungsansprüchen. Dieses Forschungs- und Darstellungsprinzip findet sich nun auch in Untersuchungen zu digitalisierten urbanen Umwelten wieder. Paul M. Torrens (2008) etwa hat Versorgungsreichweiten und Signalstärken des drahtlosen Internets (WiFi) im Stadtraum von Salt Lake City kartiert und damit Ungleichmäßigkeiten und Lücken des WiFi-Empfangs im Modus des kartographischen Darstellungsprinzips deutlich sichtbar gemacht. Christian Licoppe et al. (2008) erweitern dieses Prinzip in ihrer Studie zur Mobiltelefonie im Stadtraum von Paris um individuelle Aufenthaltsorte und Pfade konkreter Nutzersubjekte. Deren kartographische Darstellung wird mit qualitativen Informationen der Nutzer/innen kombiniert. Emmanouil Tranos (2013) folgt ebenfalls der Logik der Verortung des Technischen im Raum, operiert aber auf einer anderen Maßstabsebene. Er bietet eine Kartographie des europäischen Internets an, indem er die Signalströme zu Netzen mit unterschiedlich dicken Verbindungslinien zusammenfügt, sodass sich Konzentrationen und damit Zentren und Peripherien zeigen, die für die Nutzer/innen zumeist unsichtbar bleiben. Damit gibt er auch Hinweise auf Potentiale und Hindernisse der regionalen Entwicklung in Europa.

Indem die vertraute kartographische Darstellung angewendet wird, suggerieren diese Arbeiten, dass Technik, Kultur, Infrastruktur und das Digitale insgesamt im Raum enthalten sind. Diese Methodologie befördert die Annahme, dass das Digitale hauptsächlich in realen, materiellen Objekten existiert und dass diese einen Vorrang vor nicht-materiellen Aspekten des Digitalen aufgrund ihrer scheinbar „harten Evidenz" genießen. Die wichtigen, weil oft nicht sichtbaren materiellen „Rückseiten“ des digitalisierten Alltags bilden aber eben nur eine Facette der Geographien digitaler Kultur. Adams' Schema folgend, ordnet sich diese Form der Forschung in die „media in space“-Kategorie (Adams 2010: $44 \mathrm{ff}$.) ein oder zeigt im Sinne räumlicher Ungleichverteilungen Geographien "produced by the digital" nach Ash et al. (2016: $5 \mathrm{ff}$.) auf.

Empirische Studien reproduzieren das Schema „Technik im Raum“ aber nicht nur auf der technischmethodischen Ebene durch klassische Kartierung. Auch auf der Ebene der kritischen Deutung der Geographien des Digitalen findet sich diese Denkweise wieder, zum Beispiel, indem Figuren der unbemerkten Ausbreitung digitaler (Überwachungs-) Technik im Stadtraum konstruiert werden. Hier (z. B. bei Thrift \& French 2002; Graham 2005) wird ein Bild der Invasion gezeichnet, welches die Grundvorstellung der räumlichen Ausdehnung und Dominanz mit Blick auf das Digitale reproduziert - kritisch wird eine schleichende Ausbreitung und Dominanz des Digitalen im Raum konstatiert. Auf dieser Ebene der Betrachtung könnte es aussichtsreich sein, das - grundsätzlich natürlich berechtigte - Interesse der Geographie am Materiellen nicht nur in kartographische Repräsentationen und Invasionsfiguren zu übersetzen, sondern auch den „material turn“ in den Medienwissenschaften zu beachten. Dort werden materielle Infrastrukturen (etwa Server-Zentren in globalen Peripherien, Unterseekabel etc.) zunehmend mit kulturwissenschaftlichen und ethnographischen, statt rein kartographischen Methoden, untersucht (vgl. beispielhaft: Parks \& Starosielski 2015; Holt \& Vondereau 2015; Starosielski 2015b).

\subsection{Subjektbezogene Geographien des Digitalen}

Diese ethnographische, an Praxiskontexten und konkreten Lebenswelten interessierte Forschung deutet bereits auf eine neue Perspektive hin: die Rekonstruktion subjektiver Wahrnehmungen und individueller Aneignungen des Räumlichen. In dieser Perspektive werden Räumlichkeiten als subjektive Konstruktionen aufgefasst, die entsprechend einen verstehenden, nachvollziehenden Zugang erfordern. Orte und Räume sind das Ergebnis subjektiver Wahrnehmung und Erfahrung. Sie sind in ihrer Existenz abhängig von Bedeutungsbelegungen und Alltagspraktiken (vgl. Werlen 2008: 275 ff.; Sterly 2018: 54 ff.). So sind in den letzten Jahren einige Studien erschienen, welche die Beziehungen zwischen Subjekt und digitaler (Geo-) Information erforschen. Digitale Medien bieten neue Möglichkeiten der Verschmelzung (oder mindestens Kombination) von räumlicher Wahrnehmung, digitalem Medieninhalt und der aktiven Einbeziehung der Nutzer/innen. Zum Beispiel hat Mei-Po Kwan (2007) die individuelle (Raum-) Wahrnehmung einer musli- 
mischen Frau in den USA untersucht, indem sie diese mit digitaler Videotechnik und GPS-Gerät auf ihren alltäglichen Wegen begleitet hat. Dabei wurden die empfundenen und realen Einschränkungen der Alltagswahrnehmung und der Alltagspraxis der Frau deutlich, wie sie für die Ära nach dem 11. September typisch waren und teilweise wieder sind. Mit dem Stil dieser Studie führt Kwan die Verbindung von wissenschaftlicher Analyse, einer politischen, kritischen Haltung und der Empathie gegenüber dem Forschungssubjekt vor. Sie zeigt, dass digitale Technologien und Medien die qualitative Feldforschung bereichern können und dass sie auch neue Wege der Kommunikation zwischen Forscherin und „Beforschter" etablieren helfen.

Paul Kingsbury und John Paul Jones (2009) haben die kritische, oft auf Macht und Kontrolle konzentrierte Sicht auf digitale Geomedien erweitert, indem sie deren affektive, ästhetische und spielerische Seite betont haben. Mit Friedrich Nietzsches Unterscheidung des Apollinischen (rational, macht- und zielorientiert, strategisch) und Dionysischen (rauschhaft, gefühlsorientiert) zeigen sie am Beispiel des Geo-Browsing, dass Affekt und Emotionen (das Dionysische Element) prägend für den Umgang mit dem Medium sind. Gleichzeitig zeigen sie mit ihrer Argumentation, dass Phänomene digitaler Geographie gewinnbringend mit klassischen, philosophisch-theoretischen Begriffen aufgeschlossen werden können. Pablo Abend und Francis Harvey (2017) betonen in ihrer Analyse der Interaktion mit Geobrowsern ebenfalls deren subjektive und affektive Komponente. Sie spezifizieren diesen Aspekt, indem sie die subjektiven Unterschiede des Umgangs mit Geomedien und deren Individualisierungsmöglichkeiten beleuchten. Mark Graham et al. (2013) zeigen mit Bezug auf mehrere empirische Beispiele, dass die Digitalisierung urbaner Umwelten nicht nur von materiellen Infrastrukturen, Hardware und technischen Zugangsmöglichkeiten abhängt, sondern ebenso von den individuellen Fähigkeiten und dem Wissen ihrer Bewohner/innen, den von ihnen wahrgenommenen Handlungsoptionen und ihrer Integration in sozialen Netzwerken.

\subsection{Die soziale und kulturelle Konstruktion digitaler Räumlichkeit}

Die überindividuelle, gesellschaftlich-kulturelle Ebene wird in Arbeiten beleuchtet, die sich mit den sozialen und räumlichen Ungleichheiten im Hinblick auf den Zugang zu digitalen Medien beschäftigen. Räumliche Ausformungen des so genannten „digital divide“ bleiben auch und gerade nach der massiven, weltweiten Verbreitung digitaler Medien relevant. Auch ist die politische, staatliche Organisation digitaler Überwachung hier zu beachten, ebenso wie die Wirkmächtig- keit geographischer Imaginationen in weltweiten Diskursen auf der Ebene der Medieninhalte. So werden mit digitalen Mitteln etwa kulturräumliche Kollektive gleichermaßen als Zielpublikum wie auch als Protagonisten politischer Praxis konstruiert. Auch kann man erkennen, wie „location-based information services“ für soziale Netzwerke und lokale Gemeinschaften eine immer größere Bedeutung erlangen, gestützt durch freiwillig von Laien generierte Geoinformation (,volunteered geographic information", VGI). Vielfältige Innovationen im Bereich der „neogeography“ und des „geoweb“ erweitern zudem prinzipiell die Möglichkeiten der politischen Teilhabe. Diese neue Medien- und Kulturlandschaft verlangt nach intensiver geographischer Forschung und Kritik. Insbesondere im Bereich der "location-based services" (LBS) ${ }^{2}$ und der Integration von Geoinformation in soziale Medien und Smartphone-Apps ergeben sich neue Synthesemöglichkeiten aus digitalen Medienplattformen, erdräumlicher Mobilität und nutzergenerierten Daten. Dabei überschreiten diese neuen Möglichkeiten bereits den Fokus auf die Vermessung des einzelnen Nutzersubjektes (siehe Abschnitt 3.3) und fördern den Aufbau von Netzwerken und sozialen Gemeinschaften, die sich zunehmend auch über digitale Geoinformation im „Nahraum“ realer Lebenswelten formieren. Dies geschieht in Ergänzung und enger Beziehung zu Faceto-Face-Kontakten. Mögliche soziale Effekte wären hier eine neue Sensibilität für die Nachbarschaft als Gemeinschaft in urbanen, eigentlich anonymen Umgebungen (z. B. das Nachbarschaftsportal „nebenan. de") oder die Stärkung lokaler und regionaler Identitäten (Evans 2015; Farman 2012: 56 ff.). Für viele Menschen bildet genau diese Perspektive von Teilhabe und Kontakt eine Motivation zur Generierung und Verbreitung von VGI (Goodchild 2007; Poorthuis \& Zook 2014). Projekte wie Open Street Map (OSM) verwischen dabei die Grenzen zwischen professioneller und Laienkartographie, stimulieren freiwilliges Nutzer/innenengagement als gemeinschaftliche Aktivität und helfen, passive Konsument/innen in aktive „Prosumer" oder „Produser“ zu verwandeln. Aktuell wird dieses Engagement allerdings nur von (relativ!) Wenigen realisiert - nur $5 \%$ der Nutzer von OSM generieren regelmäßig Inhalte; $95 \%$ der Nutzer/innen bleiben letztlich passive Konsument/innen (vgl. Neis \& Zipf 2012). Bei der aktiven Teilhabe zeigt sich ein deutliches Geschlechterungleichgewicht zugunsten der männlichen Mitglieder, was sich zum Teil auch in Karteninhalten widerspiegelt (Stephens 2013). Auch

Dabei handelt es sich um GPS-basierte, standortbezogene Dienste, die beispielsweise der Information und Navigation im Stadtraum und dem Auffinden so genannter POIs (points of interest) dienen können. 
verfestigen die Dynamiken der Selbstorganisation in OSM tendenziell Eliten erfahrener Nutzer mit entsprechendem Einfluss auf die Gestaltung der Plattform und die Selektion der Inhalte (Elwood 2010; Perkins 2014; Haklay 2013). Aktuell jedenfalls ist diese relativ kleine Gruppe soziologisch noch sehr homogen - die Schwerpunktgruppe der produktivsten Mitglieder ist meist westlich, männlich, jung, weiß und gebildet. Auch ethnische, städtische Segregation bildet sich bisweilen sehr deutlich in unterschiedlichen Kartierdichten ab (Bittner 2014). Trotzdem bieten diese Plattformen für die Zukunft einen potentiell breiteren Horizont der Partizipation. Dieser muss zunächst einmal prinzipiell eröffnet werden, denn viele Anwendungen, die aktuell mit Geodaten operieren, „ernten“ diese lediglich auf automatisierte Weise von den Geräten von Endnutzer/innen, die bestenfalls einmalig diesen Datenübertragungen zugestimmt haben, aber genau keine selbstbestimmte, aktive Beteiligung an deren Verarbeitung und Kommunikation erlangen (Harvey 2013). Francis Harvey (ebd.) plädiert deshalb für eine engere Definition von VGI, die sich auf die bewusste und selbstbestimmte Produktion und Verarbeitung von Geoinformation beschränkt.

Die Widersprüchlichkeit neuer Geomedien wird auch in Lisa Parks' Betrachtungen zur Krisenkartierung in Google Earth deutlich (Parks 2009). Am Beispiel der Darfur-Krise zeigte sie Potentiale der digitalen Geovisualisierung auf, die sowohl der Aufklärung als auch der Manipulation der Weltöffentlichkeit dienen können. Die Beziehungen zwischen der westlichen, tradierten geographischen Vorstellungswelt, scheinbar neutralen Geoinformationen, deren politischer Deutung, der Information der Öffentlichkeit und den Möglichkeiten zur Diskurssteuerung werden hier offenkundig.

Was Forschungen zur soziokulturellen Konstruktion digitaler Räumlichkeit interessant macht, ist die zunehmende Sensibilität für den Facettenreichtum der sozialen Effekte des Digitalen. Zum Beispiel wird die Frage nach Fortbestand oder Wandel der vielfältigen Geographien der digitalen Kluft zunehmend differenzierter diskutiert. Nicht nur Zugangsmöglichkeiten zu digitalen Infrastrukturen werden betrachtet, sondern auch die realen Freiheiten ihrer Nutzung, die Unterschiede, die in kulturellen Kontexten und Präferenzen wurzeln, Geschlechterunterschiede und ungleich verteilte Bildungschancen im Zusammenhang mit der Digitalisierung. Gleichzeitig müssen aber auch die Kategorien, mit denen diese Gegensätze typischerweise gerahmt werden, angesichts des schnellen sozio-technischen Wandels beständig neu geprüft und angepasst werden. Die Rolle klassischer Autoritäten ändert sich, die Bedeutung von Expert/innenwissen wird neu bestimmt und die Modi der Arbeitsteilung werden umgeformt - im Bereich der Bildung im Allgemeinen und der Kartographie und Geographie im Besonderen. Wenn Laien und informelle Kollektive digitale Technologie entwickeln und verbreiten helfen, müssen entsprechend neue Begriffe von Wissen, Arbeit, Macht und Raum auch diese flüchtigeren, oft nischenhaften Phänomene mit in den Blick nehmen.

Auch die widersprüchlichen Befunde zur Rolle mobiler digitaler Medien für die Geographien des Alltags sind zu reflektieren. Sie vermitteln manchmal das Gefühl, dass Präsenz, Unmittelbarkeit und die Bedeutung unserer physischen Umgebung verblassen. Gleichzeitig bieten so genannte „Augmented Reality“Anwendungen neue Möglichkeiten, Kontakte zu unserer physischen Umgebung aufzunehmen, indem sie ortsbezogene Informationen liefern und damit den Ort mit Bedeutung anreichern, ja sogar neue soziale Netzwerke und Gemeinschaften formen helfen können. Hierzu sei an die bekannte Formel der "Glokalisierung" (Robertson 1995) erinnert, mit der die bleibende Signifikanz lokaler Informationen im globalisierten Zeitalter betont wird. Gerade in Zeiten, in denen verschiedenste Lebenswirklichkeiten (Kulturspezifika, politische Verhältnisse, Konsumoptionen etc.) global vergleichbar und erreichbar sind, bleiben Geographien des Digitalen auch im Sinne global verbreiteter, aber eben genau nicht identischer geographischer Imaginationen erhalten. Medien formen unsere Weltsicht, und so werden auch digitale Medien geographische Imaginationen auf neuen Wegen konstruieren.

\section{Digitale Alltagskultur, Unterricht und Schule}

Mit dem Aufschwung digitaler Alltagskultur als Thema in der (geographischen) Fachwissenschaft ist nun die Frage verbunden, wie sich die skizzierten Überlegungen auf die Unterrichts- bzw. Schulpraxis beziehen lassen. Zwei Ansätze bzw. Blickrichtungen bieten sich dabei an: Einerseits können die Geographien des Digitalen als Inhalt des Lehrens und Lernens (weiter) Eingang in den GW-Unterricht finden. Andererseits jedoch kann die Schulpraxis selbst als Form digitaler Alltagskultur in den Fokus genommen werden.

\subsection{Leitkonzepte und Themenbeispiele für den GW-Unterricht}

Der Anspruch, das Digitale nicht nur als Teil didaktischer Konzepte und Praktiken zu fassen, sondern es selbst als Inhalt im GW-Unterricht zu thematisieren, wirft zuerst die Frage auf, wie sich Geographien des Digitalen von allgemeinen, fächerübergreifenden 
Themen der Digitalisierung unterscheiden. Die im vorherigen Abschnitt beschriebenen Forschungsfelder der wissenschaftlichen Geographie liefern dazu wichtige Anregungen - sowohl für die theoretisch-konzeptionelle und didaktische Anleitung der Lehrenden als auch für die Auswahl konkreter Lehrinhalte für die Lernenden.

So können für die Lehrenden (und deren Ausbildung) zunächst Fragen der theoretischen Aufgliederung des Themas hilfreich sein. Paul Adams' (2010; siehe Abschnitt 3.1) Grundunterscheidung zwischen Medien im Raum und dem Raum in den Medien liefert beispielsweise eine erste logische Orientierung für die thematische Ordnung im Unterricht. Ähnlich wäre der von James Ash et al. (2016; siehe Abschnitt 3.1) unterbreitete Vorschlag einer Unterscheidung zwischen digitaler Geographie im Sinne (a) ihrer methodisch-technischen Modernisierung („geographies through the digital"; etwa GIS im GW-Unterricht), (b) der erdräumlich ungleich verteilten Folgen und Phänomene der digitalen Revolution (,geographies produced by the digital"), und (c) den alltagsweltlich und individuell neu arrangierten Raumbezügen im Zuge der Digitalisierung (,geographies of the digital“) $\mathrm{zu}$ verwenden. Darüber hinaus sind auch generelle Modelle zur Integration von Geoinformation - sowohl in die Lebenswelt als auch in den Unterricht - von besonderem Nutzen, die bereits als Klammer zwischen Fachwissenschaft, Didaktik und angepeilter Gesellschaftsrelevanz konzipiert wurden (vgl. Gryl \& Jekel 2012).

Auf der Ebene konkreter empirischer Gegenstände könnten sich zwei Arten von Themen für die Schüler/innen als besonders geeignet erweisen: Zum einen Themen, die klar der Verortungslogik der klassischen Geographie folgen (Modus „Technik im Raum"). Hier geht es um das Aufzeigen räumlicher Ungleichverteilungen des Digitalen, in denen sich letztlich auch soziale Ungleichheiten spiegeln. Karten bleiben hier das dominante didaktische Mittel, um Vernetzungen (oder deren Fehlen) und Regionalisierungen sichtbar zu machen. Die vielfältigen Formen einer solchen „digitalen Kluft“ („digital divide“, siehe stellvertretend Warf 2018) reichen von Konzentrationen der materiellen Infrastruktur (z. B. Server-Zentren im subarktischen Raum oder Unterseekabel für die globale Kommunikation) ${ }^{3}$, Agglomerationen der ökonomischen Wertschöpfung und Kontrolle (z.B. Silicon-Valley), Clustern digitaler Dienstleistungen (z. B. Callcenter in Indien) über daran anschließende, weltweit ungleich verteilte Zugangsmöglichkeiten mit

\footnotetext{
Siehe dazu: Starosielski (2015b); Malecki \& Wei (2009) und für aktuelle Kartographien: https://www.submarinecablemap.com (zuletzt abgerufen am 27.01.2019).
}

einer andauernden Benachteiligung des globalen Südens. Auch auf lokaler und regionaler Maßstabsebene (vgl. Torrens 2008; s. o.) zeigen beispielsweise Lücken in der Versorgung mit schnellem Internet abgemilderte Formen der digitalen Kluft, die für die Schüler/ innen greifbar sind. Zudem können kartographische Visualisierungen der weltumspannenden Signalwege der eigenen Suchabfragen im Internet (z. B. mit dem Tool „VisualRoute“) die erdräumlichen Bezüge des Digitalen für die Lernenden aufzeigen (vgl. Starosielski 2015b; Malecki \& Wei 2009; Telegeography 2019). Damit wird die Black Box digitaler Systeme durchsichtiger - jenseits der für Schüler/innen allgegenwärtigen Screens und Benutzeroberflächen. Der kartographischen Logik folgend wird das Digitale hierbei als räumliches Objekt vorgestellt. So limitiert dieser Zugang aus fachtheoretischer Sicht erscheint, so erfolgreich ist er als alltägliches Deutungsmuster. Die Vorstellung von Geographie als räumliche Verortung von Dingen ist im Alltagsleben fest etabliert und logisch einfach bzw. einfach logisch. Er ist deshalb didaktisch dankbar, weil das Digitale mit Hilfe des Prinzips Karte transformiert und gleichzeitig leicht wiedererkannt wird.

Die Vermittlung der Geographien des Digitalen im GW-Unterricht sollte sich aber natürlich nicht auf diese Ebene beschränken. Im zweiten Schritt sollten Themen gewählt werden, die möglichst nah an der Lebenswelt der Lernenden angesiedelt sind. Neben dem Schritt der Verteilung oder Verortung sollte die alltagspraktische Welt-Bindung in den Mittelpunkt rücken. Konkrete Lebenswelten und deren digitale Transformation können etwa anhand von Beispielen aus anderen Weltgegenden illustriert werden. So hat Harald Sterly (2018) das Mobiltelefon als Mittel der Aufrechterhaltung familiärer Beziehungen über Distanz im ländlichen Bangladesh (Arbeit in der Stadt Familie auf dem Land) untersucht. Die qualitativen Interviewausschnitte geben unmittelbare Einblicke in den Alltag der Menschen und die Rolle digitaler Technik für dessen raumzeitliche Strukturierung. Ein noch stärkerer Anwendungs- und Übungsbezug lässt sich mit lokativen Medien herstellen, die eine immer größere Bedeutung im Alltag der Lernenden erlangen (z. B. das Suchen und Finden von Orten in der Umgebung; die Navigation im Stadtraum). Daran lässt sich mit Übungen zum partizipativen Kartieren (Bsp. Open Street Map; s. o.) anschließen. Fortgeschrittene Lernende können auch zu einer kritischen Reflexion der sozialen Voraussetzungen und Wirkungen von „volunteered geographic information" eingeladen werden (Was wird kartiert? Von wem? Was fehlt auf den Karten?). So können letztlich kritische Diskussionen zu den in die Alltagspraktiken eingelassenen Machtbeziehungen mit Bezug auf alle räumlichen 
Maßstabsebenen, vor allem aber auch mit Bezug auf die alltäglichen Routinen der Lernenden angeregt werden. Unabhängig von den gewählten Einzelthemen gilt es, sich mit dem Begriff des Digitalen - mit einem kritischen Blick auf gegenwärtige Erzählungen - auseinanderzusetzen.

\subsection{Digitale Alltagskultur und Schule}

Die mit dem Alltagsbezug der skizzierten Themen eingenommene Lebenswelt-zentrierte Perspektive kann nun natürlich nicht nur auf schulexterne Kontexte angewendet werden, sondern selbstverständlich auch auf Schule als digitalisierte Lebenswelt selbst. Auch der Schulalltag - in all seinen Aspekten - ist von Digitalisierungsprozessen nicht ausgenommen und wird damit zum potenziellen Reflexionsgegenstand. Das entspricht auch der alltäglichen Erfahrung: Lehrende kommunizieren per Messenger-App, Schüler/ innen nutzen soziale Netzwerke und arbeiten zusammen an Online-Dokumenten, Daten werden auf Servern gespeichert und geteilt, Geodatenportale werden zur Planung von Exkursionen genutzt usw. Digitale Alltagskultur durchdringt ganz ohne systematische Anstrengungen oder Planungsmaßnahmen die alltägliche Gestaltung von Schule und Unterricht. ${ }^{4}$ Mit dieser schleichenden Digitalisierung von Schule und Unterricht sind allerdings - wie bei allen anderen gesellschaftlichen Entwicklungen auch - für den Schulkontext elementare Fragen nach einheitlichen Standards, Unterrichtsprinzipien, Ausbildungsinhalten, Kompetenz- und allgemeiner Professionsentwicklung verbunden, die nicht nur bildungswissenschaftliche und organisationstheoretische Expertise verlangen, sondern zunächst einmal ein grundlegendes Verständnis des Phänomens Digitalisierung.

Angebote, die mit der Digitalisierung von Schule und Unterricht verbundenen Herausforderungen zu systematisieren, finden sich gegenwärtig vor allem in den in zahlreichen Ländern entwickelten Kompetenzmodellen für digitale Bildung. So etwa wurden im 2014 in der Schweiz verabschiedeten "Lehrplan 21" (vgl. D-EDK 2016) differenzierte Schlüsselkompetenzen zur digitalen Mündigkeit in den Bereichen Medien und Informatik ausgewiesen. Im deutschen Kontext gibt es seit 2016 ein von der Kultusministerkonferenz (KMK) im Rahmen der Strategie „Bildung in der digitalen Welt" entwickeltes Handlungskonzept, das Rahmenkompetenzen

\footnotetext{
${ }^{4}$ Auch wenn hier einschränkend natürlich einerseits betont werden muss, dass in Schule, als besonderem Handlungskontext, digitale Technologien kaum unreflektiert übernommen werden, und dass sich andererseits unter dem Sammelbegriff „Schule“ ganz verschiedene pädagogische Konzepte - mit teilweise sehr unterschiedlichen Auffassungen in Bezug auf digitale Medien - finden können.
}

zum Lehren und Lernen in der digitalen Welt definiert (vgl. KMK 2016). In Österreich schließlich gibt es seit 2016 das im Kontext der Digitalstrategie des Bundesministeriums für Bildung $(\mathrm{BMB})$ von der Virtuellen PH erstellte Kompetenzmodell „digi. kompP" (vgl. Brandhofer et al. 2016), das wir hier aufgrund seines umfassenden Charakters exemplarisch herausgreifen möchten.

Im „digi.kompP“-Kompetenzmodell werden die Kompetenzen für einen reflektierten Umgang mit digitalen Technologien im Schulalltag - zumindest für Lehrer/innen - skizziert und für die Ausbildung digital souveräner Lehrender strukturiert. Das Modell umfasst dabei sowohl Kompetenzen, die während der Lehramtsausbildung erworben werden sollen als auch solche, die vor dem Studium oder während der ersten fünf Praxisjahre erworben werden sollen (siehe Abb. 2).

Modelle wie „digi.kompP“ sind für eine Auseinandersetzung mit Schule als Beispielfall digitaler Alltagskultur einerseits von Bedeutung, weil sie normative Vorstellungen davon liefern, wie digitales Alltagsleben im Schulkontext zu gestalten ist. Sie gliedern die Lebenswelt Schule analytisch in verschiedene Handlungsfelder. So unterscheidet "digi.kompP" acht Kompetenzbereiche, in denen Digitalisierungsprozesse für den Schulkontext relevant sind. Diese reichen von informatischen Grundkompetenzen (u.a. Wissen über technische Funktionsweisen von Computern und Netzwerken, die Produktion digitaler Medien und Datenschutz) über Methoden des digitalen Lehrens und Lernens (u.a. Fähigkeit zum Gestalten digitaler Lernumgebungen, Einsatz von EPortfolios und Kollaborationssystemen), bis hin zur „Digital-inklusive[n] Professionsentwicklung" (u.a. Befähigung zur digital gestützten Forschung und zum digital gestützten Selbstmanagement, oder souveräne Gestaltung der eigenen Online-Identität). ${ }^{5}$ Mit diesen Bereichen liefert das Kompetenzmodell gleichsam en passant eine feingliedrige Heuristik digitalen Schulalltags, die insbesondere deshalb hilfreich ist, weil sie Digitalisierungsfragen aus der Nische reduktionistischer Argumentationen à la „Tablets oder keine Tablets im Unterricht?" herausholt und auch auf die mit der Digitalisierung verbundenen Herausforderungen jenseits konkreter Unterrichtspraxis aufmerksam macht. So dürfte es in der Lehramtsausbildung bislang kaum systematische Lehrinhalte zur „digitale[n] Schulgemeinschaft" (Brandhofer et al. 2016: 11) oder zum „digitale[n] Verwalten“ (ebd.: 10) geben, die jedoch in mittel- und langfristiger Perspektive auch für die Schule als Teil der modernen Arbeitswelt essentiell sein dürften.

\footnotetext{
${ }^{5}$ Für eine vollständige Übersicht siehe Brandhofer et al. (2016).
} 


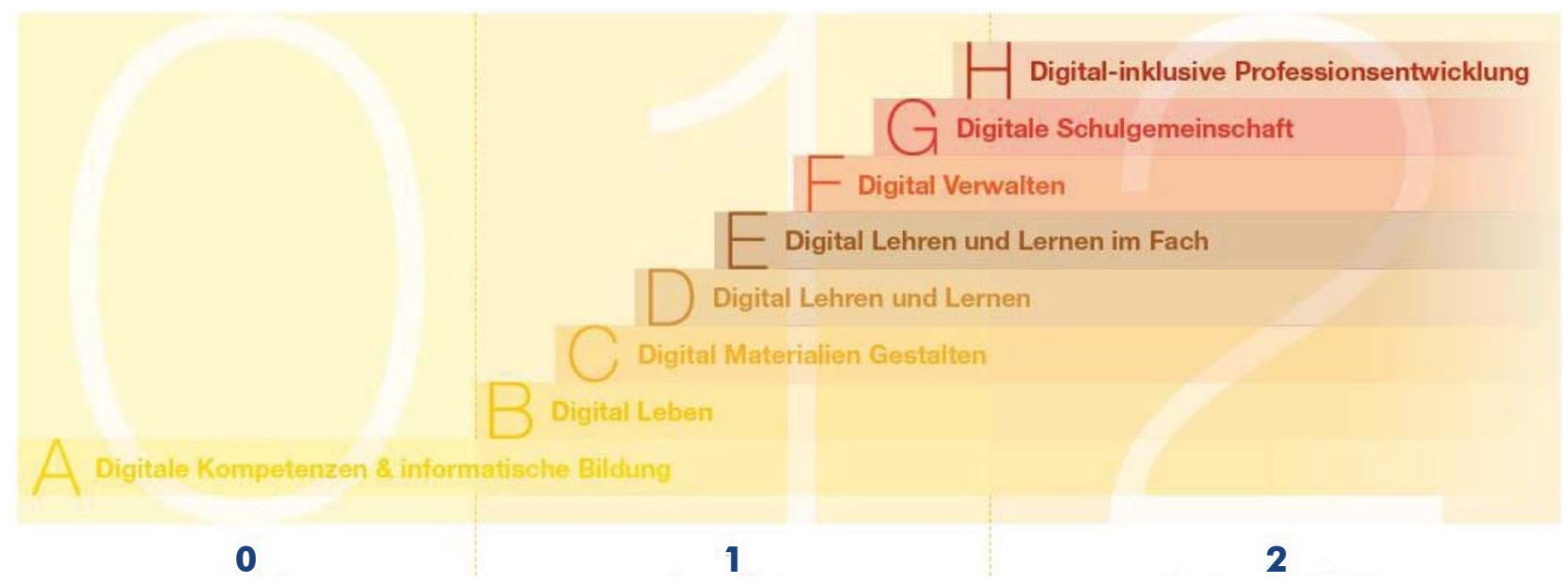

vor dem Studium

$\begin{array}{ll}\text { A } & \text { B } \\ \text { Digitale Kom- } & \text { Leben, Lehren } \\ \text { petenz und } & \text { und Lernen im } \\ \text { informatorische } & \text { Zeichen der } \\ \text { Bildung auf } & \text { Digitalität, Fragen } \\ \text { Maturaniveau und } & \text { der Technikethik, } \\ \text { dessen laufende } & \text { Medienbildung } \\ \text { Aktualisierung } & \text { und -biographie, } \\ & \text { Barrierefreiheit }\end{array}$

$\begin{array}{lll}\text { C } & \text { D } & \text { E } \\ \text { Gestalten, } & \begin{array}{l}\text { Planen, Durchfüh- } \\ \text { Verändern und } \\ \text { ren und Evaluie- }\end{array} & \begin{array}{l}\text { Fachspezifisch } \\ \text { lernförderlicher }\end{array} \\ \text { Veröffentlichen } & \text { ren von Lehr- und } & \text { Einsatz von } \\ \text { von Unterlagen } & \text { Lernprozessen mit } & \text { Content, Software, } \\ \text { für den Unterricht; } & \text { digitalen Medien } & \text { Medien und Werk- } \\ \text { Rechtsfragen und } & \text { und Lernumge- } & \text { zeugen } \\ \text { damit verbunde- } & \text { bungen; formative } & \\ \text { ne Rechte und } & \text { und summative } & \\ \text { Pflichten (Creative } & \text { Beurteilung; } \\ \text { Commons, Werk- } & \text { Feedback; Safer } \\ \text { nutzung, Úrheber- } & \text { Internet } \\ \text { recht, OER) } & \end{array}$

während des Studiums während der ersten fünf Praxisjahre

Abb. 2: Das „digi.kompP“-Kompetenzmodell (Brandhofer et al. 2016: 1)

Neben ihrem instruktiven Charakter sind Kompetenzmodelle wie „digi.kompP“ für eine sozial- und kulturwissenschaftlich fundierte Auseinandersetzung mit Digitalisierungsprozessen jedoch auch noch aus einem zweiten Grund von Interesse. Begreift man die Modelle nämlich nicht nur als schlichtweg gegebene Regularien zur Gestaltung der Lebenswelt Schule, sondern, aus reflexiver Distanz heraus, auch als Ergebnisse einer bestimmten Sicht auf die Digitalisierung, dann lassen sie sich auch als Zeitdokument lesen, als Ausdruck eines bestimmten, historisch situierten Verständnisses des Digitalen und der damit verbundenen Gestaltungsspielräume und Risiken.

Eine solche Perspektive auf Dokumente wie das Kompetenzmodell „digi.kompP“ würde - von Grundsatzfragen wie dem Digitalisierungsverständnis einmal ganz abgesehen - beispielsweise anhand von Formulierungen wie „Wechselwirkung zwischen Technologie und Gesellschaft" (Brandhofer et al. 2016: 6) oder „Einfluss von Informatiksystemen auf meinen Alltag" (ebd.: 3) die impliziten techniksoziologischen Positionen hinter dem Modell rekonstruieren und auf Widersprüche oder bislang ungeklärte Verhältnisse aufmerksam machen. Darüber hinaus ließen sich die gegenwärtigen Leitthemen der Digitalisierung - wie etwa Modellierung und Simulation, die Rolle von Algorithmen und sozialen Netzwerken oder Fragen des Datenschutzes (Brandhofer et al. 2016: 5, 6, 12) - identifizieren und blinde Flecken des Modells, wie etwa die materielle Basis digitalen Unterrichtens, ausfindig machen. Und nicht zuletzt könnte eine kritische Inblicknahme auch auf vermeintlich themenfremde, aber für die Sozial- und Kulturwissenschaften nichtsdestotrotz zentrale Argumentationsfiguren aufmerksam machen, wie etwa die im Dokument aufscheinende Gegenüberstellung von Gesellschaft und Wirtschaft (vgl. z. B. Brandhofer et al. 2016: 3).

Einer an solchen Themen orientierten Auseinandersetzung darf es dabei selbstverständlich nicht um bloße Dokumentenkritik gehen. Vielmehr dokumentiert eine kritische und offen geführte Debatte um die Digitalisierung der Lebenswelt Schule einen souveränen Umgang - nicht nur mit digitalen Technologien, sondern eben auch mit den sie begleitenden Regularien und Empfehlungen. Hierin unterscheidet sich der Lebenskontext Schule nicht von Praktiken wie den oben skizzierten, digitale Mündigkeit erfordernden Formen digitalen Geographie-Machens.

\section{$5 \quad$ Fazit}

Mit Blick auf die aktuellen bildungspolitischen Debatten wird deutlich, dass die zunehmende Verbrei- 
tung und Nutzung digitaler Technologien Schule und Unterrichtspraxis mit neuen Herausforderungen konfrontiert. Diese sind dabei nicht nur technischer Art, wie im öffentlichen Diskurs häufig dominant, sondern betreffen insbesondere Unterrichtsinhalte - wie wir am Beispiel der Geographien des Digitalen zu illustrieren versucht haben - sowie den Modus des Unterrichtens und des alltäglichen Gestaltens von Schule.

Bei der Bearbeitung dieser Herausforderungen kann es hilfreich sein, Schule trotz ihrer Spezifika nicht in erster Linie als spezielles Feld zu betrachten, sondern zunächst einmal als Teil einer weiter gefassten digitalen Alltagskultur. Damit wird einerseits der Blick für die vielen bereits vorhandenen Kompetenzen und das bei Lehrenden sowie Schüler/innen oft schon existierende Problembewusstsein geöffnet. Nicht alles was mit Digitalisierung zu tun hat, muss im Schulkontext neu gelernt werden. Zum anderen jedoch erlaubt eine solche Perspektive auch die Repositionierung des Digitalen im schulischen Kontext. Wie in anderen Lebensbereichen auch, verändert die Digitalisierung nicht schlagartig die Logik der Alltagspraxis von Grund auf, sondern das Digitale schließt an vielen Stellen an - zum Teil erstaunlich widerständige „analoge“ Praktiken an. Dies zeigt sich gleichermaßen an geographischen wie nicht-geographischen Fällen.

Dasselbe Argument lässt sich schließlich auch auf die Inhalte des Unterrichts beziehen. Digitale Alltagskultur als Gegenstand des GW-Unterrichts mag auf den ersten Blick ein völlig neues Themenfeld darstellen. Wie die obigen Ausführungen zu den Geographien des Digitalen aber gezeigt haben, schließen diese an vielen Stellen an eingeübte geographische Denkmuster und Theoriefiguren (wie etwa die Frage nach der räumlichen Verteilung von Infrastrukturen, Praktiken des Kartierens oder die mediale Repräsentation von Orten) an. Eine besondere Sensibilität für diese Spannung von Kontinuität und Diskontinuität - und damit die Ambivalenz der digitalen Wende insgesamt - zu entwickeln, könnte eine Stärke des GW-Unterrichts sein.

\section{Dank}

Die Veröffentlichung dieses Beitrags wurde vom Open Access Publikationsfonds der Universität Jena unterstützt.

\section{Literatur}

Abend, P. \& F. Harvey (2017): Maps as geomedial action spaces: considering the shift from logocentric to egocentric engagements. In: GeoJournal 82(1). S. 171-183. http://dx.doi.org/10.1007/s10708-015-9673-z (zuletzt abgerufen am 29.01.2019).

Adams, P. (2009): Geographies of media and communication. Wiley \& Blackwell, Malden, MA.

Adams, P. (2010): A taxonomy for communication geography. In: Progress in Human Geography 35(1). S. 37-57.

Alizadeh, T., N. Sipe \& J. Dodson (2014): Spatial planning and high-speed broadband: Australia's national broadband network and metropolitan planning. In: International Planning Studies 19(3-4). S. 359-378.

Amoore, L. \& V. Piotukh (2016) (Hrsg.): Algorithmic life: calculative devices in the age of big data. Routledge, New York.

Ash, J., R. Kitchin \& A. Leszczynski (2016): Digital turn, digital geographies? In: Progress in Human Geography, Online first. https://doi.org/10.1177/0309132516664800 (zuletzt abgerufen am 29.01.2019)

Barkhoff, J., H. Böhme \& J. Riou (2004) (Hrsg.): Netzwerke: Eine Kulturtechnik der Moderne. Böhlau, Köln.

Beck, U. \& E. Beck-Gernsheim (2011): Fernliebe. Lebensformen im globalen Zeitalter. Suhrkamp, Berlin.

Berghel, H. (1997): Email: the good, the bad, and the ugly. In: Communications of the ACM 40(4). S. 11-15.

Berry, D. M. (2013): Critical theory and the digital. Bloomsbury, New York.

Bittner, C. (2014): Reproduktion sozialräumlicher Differenzierungen in OpenStreetMap: das Beispiel Jerusalems. In: Kartographische Nachrichten 64(3). S. 136-143.

Blatt, A. J. (2015): The benefits and risks of volunteered geographic information. In: Journal of Map and Geography Libraries, 11(1). S. 99-104. http://dx.doi.org/1 0.1080/15420353.2015.1009609 (zuletzt abgerufen am 29.01.2019).

Brandhofer, G., A. Kohl, M. Miglbauer, T. Nárosy, J. Buchner, P. Groißböck, I. Lechner, J. Prinz, J. Prohaska, N. Zaynard, W. Fikisz, G. Futschek, K. Fuchs, P. Micheuz, H. Caba, W. Grossmann, A. Nußbaumer, G. Schwarz, F. Tranninger, R. Zoufal, H. Hammerl, H. Egger, H. Swaton, M. Steiner, A. Bachinger, S. Gabriel, C. Nosko, M. Schedler, P. Traxler, W. Wegscheider \& D. Wohlhart (2016): Das digi.kompP Kompetenzmodell. https:// www.virtuelle-ph.at/wp-content/uploads/2016/09/digi. kompP-Grafik-und-Deskriptoren-1.pdf (zuletzt abgerufen am 29.01.2019)

Brennen, J. S. \& D. Kreiss (2016): Digitalization. In: Bruhn Jensen, K. \& R. T. Craig (Hrsg.): The international encyclopedia of communication theory and philosophy, Volume I: A-D. Wiley Blackwell, Chichester. S. 556-566.

Brynjolfsson, E. \& A. McAfee (2011): Race against the machine: how the digital revolution is accelerating innovation, driving productivity, and irreversibly transforming employment and the economy. Digital Frontier Press, Lexington, Massachusetts.

Brynjolfsson, E. \& A. McAfee (2014): The second machine age: work, progress, and prosperity in a time of brilliant technologies. W. W. Norton, New York. 
Burckhardt, M. (2017): Eine kleine Geschichte der Digitalisierung. In: Merkur 71(816). S. 47-61.

Byington, K. W. \& D. C. Schwebel (2012): Effects of mobile internet use on college student pedestrian injury risk. In: Accident Analysis and Prevention 51. S. 78-83. https://doi.org/10.1016/j.aap.2012.11.001 (zuletzt abgerufen am 29.01.2019).

Castells, M. (2001): Der Aufstieg der Netzwerkgesellschaft (= Das Informationszeitalter Bd. 1). Leske + Budrich, Opladen.

Couldry, N. (2012): Media, society, world: social theory and digital media practice. Polity Press, Cambridge.

Dodge, M. \& R. Kitchin (2005): Code and the transduction of space. In: Annals of the Association of American Geographers 95(1). S. 162-180.

Dolata, U. (2013): The transformative capacity of new technologies: a theory of sociotechnical change. Routledge, London.

Dyer-Witheford, N. (2015): Cyber-proletariat: global labour in the digital vortex. Between the Lines, Toronto.

D-EDK [Deutschschweizer Erziehungsdirektoren-Konferenz] (2016): Lehrplan 21. Von der D-EDK Plenarversammlung am 31.10.2014 zur Einführung in den Kantonen freigegebene Vorlage. Bereinigte Fassung vom 29.02.2016. www.lehrplan.ch (zuletzt abgerufen am 01.04.2019).

Elwood, S. (2010): Geographic information science: emerging research on the societal implications of the geospatial web. In: Progress in Human Geography 34(3), S. 349-357.

Evans, L. (2015): Locative social media: place in the digital age. Palgrave Macmillan, Basingstoke.

Farman, J. (2012): Mobile interface theory: embodied space and locative media. Routledge, London.

Felgenhauer, T. \& K. Gäbler (2018) (Hrsg.): Geographies of Digital Culture. Routledge, London.

Floridi, L. (2015): Die vierte Revolution. Wie die Infosphäre unser Leben verändert. Suhrkamp, Berlin.

Giessmann, S. (2014): Die Verbundenheit der Dinge: Eine Kulturgeschichte der Netze und Netzwerke. Kadmos, Berlin.

Goodchild, M. (2007): Citizens as sensors: the world of volunteered geography. In: GeoJournal 69(4). S. 211-221.

Graham, S. (2005): Software-sorted geographies. In: Progress in Human Geography 29(5). S. 562-580.

Graham, M., M. Zook \& A. Boulton (2013): Augmented reality in urban places: contested content and the duplicity of code. In: Transactions of the Institute of British Geographers 38(3). S. 464-479.

Gryl, I. \& T. Jekel (2012): Re-centering geoinformation in secondary education: Toward a spatial citizenship approach. In: Cartographica. The International Journal for Geographic Information and Geovisualization 47(1). S. $18-28$.

Haklay, M. (2013): Neogeography and the delusion of democratisation. In: Environment and Planning A 45(1). S. 55-69.
Hansraj, K. K. (2014): Assessment of stresses in the cervical spine caused by posture and position of the head. In: Surgical Technology International 25. S. 277-279.

Harvey, F. (2013): To volunteer or to contribute locational information? Towards truth in labeling for crowdsourced geographic information. In: Sui, D., S. Elwood \& M. F. Goodchild (Hrsg.): Crowdsourcing geographic knowledge: volunteered geographic information (VGI) in theory and practice. Springer, Dordrecht. S. 31-42.

Holt, J. \& P. Vondereau (2015): Where the Internet Lives. Data Centers as Cloud Infrastructure. In: Parks, L. \& N. Starosielski (Hrsg.): Signal Traffic. Critical Studies of Media Infrastructures. University of Illinois Press, Urbana. S. 71-93.

Horst, H. A. \& D. Miller (2013) (Hrsg.): Digital anthropology. Bloomsbury, London.

Horvath, R. (1974): Machine space. In: The Geographical Review 64(2). S. 166-187.

Huws, U. (2003): Cybertariat: virtual work in a real world, Monthly Review Press, New York.

Kavada, A. (2016): Social movements and political agency in the digital age: a communication approach. In: Media and Communication 4(4). S. 8-12.

Kingsbury, P. \& J. P. Jones III (2009): Walter Benjamin's Dionysian adventures on Google Earth. In: Geoforum 40(4). S. 502-513.

KMK [Kultusministerkonferenz] (2016): Bildung in der digitalen Welt. Strategie der Kultusministerkonferenz. https://www.kmk.org/fileadmin/Dateien/veroeffentlichungen_beschluesse/2018/Strategie_Bildung_in_der_ digitalen_Welt_idF._vom_07.12.2017.pdf (zuletzt abgerufen am 01.04.2019).

Kohut, A., C. Bowman \& M. Petrella (1995): Technology in the American household: Americans going online: explosive growth, uncertain destinations. http://www. people-press.org/1995/10/16/americans-going-onlineexplosive-growth-uncertain-destinations/ (zuletzt abgerufen am 29.01.2019).

Kwan, M.-P. (2007): Affecting geospatial technologies: toward a feminist politics of emotion. In: The Professional Geographer 59(1). S. 22-34.

Lagerkvist, A. (2017): Existential media: toward a theorization of digital thrownness: In: new media $\&$ society 19(1). S. 96-110.

Laurier, E. (2001): Why people say where they are during mobile phone calls. In: Environment and Planning D: Society and Space 19(4). S. 485-504.

Lesczszynski, A. (2015): Spatial media/tion. In: Progress in Human Geography 39(6). S. 729-751.

Lee, F. L. F., H.-T. Chen \& M. Chan (2017): Social media use and university students' participation in a large-scale protest campaign: the case of Hong Kong's umbrella movement. In: Telematics and Informatics 34(2). S. 457-469.

Licoppe, C., D. Diminescu, Z. Smoreda \& C. Ziemlicki (2008): Using mobile phone geolocalisation for 'sociogeographical' analysis of co-ordination, urban mobilities, and social integration patterns. In: Tijdschrift voor Economische en Sociale Geografie 99(5). S. 584-601. 
Lupton, D. (2016): The quantified self. Polity Press, Cambridge.

Maddalena, K. \& J. Packer (2015): The digital body: telegraphy as discourse network. In: Theory, Culture \& Society 32(1). S. 93-117.

Maireder, A. \& J. Ausserhofer (2014): Political discourses on Twitter: networking topics, objects, and people. In: Weller, K., A. Bruns, J. Burgess, M. Mahrt \& C. Puschmann (Hrsg.): Twitter and society. Peter Lang, New York. S. 305-318.

Malecki, E. J. \& H. Wei (2009): A wired world: The evolving geography of submarine cables and the shift to Asia. In: Annals of the Association of American Geographers 99(2). S. 360-382.

Marvin, C. (1988): When old technologies were new: thinking about electric communication in the late nineteenth century. Oxford University Press, Oxford.

Matthewman, S. (2011): Technology and social theory. Palgrave Macmillan, New York.

Miller, V. (2011): Understanding digital culture. Sage, London.

Minsky, M. (1980): Telepresence. In: Omni 2(9). S. 45-52.

Morell, J. A. (1988): The organizational consequences of office automation: refining measurement techniques. In: ACM SIGMIS Database 19(3-4). S. 16-23.

National Academy of Engineering (1983): The long-term impact of technology on employment and unemployment. National Academy Press, Washington, D. C.

Neff, G. \& D. Nafus (2016): Self-tracking. MIT Press, Cambridge, MA.

Neis, P. \& A. Zipf (2012): Analyzing the contributor activity of a volunteered geographic information project: the case of OpenStreetMap. In: ISPRS International Journal of Geo-Information 1(2). S. 146-165.

O'Neil, C. (2016): Weapons of math destruction: how big data increases inequality and threatens democracy. Crown, New York.

Osterhammel, J. (2009): Die Verwandlung der Welt. Eine Geschichte des 19. Jahrhunderts. C. H. Beck, München.

Park, D. W., N. W. Jankowski \& S. Jones (2011) (Hrsg.): The long history of new media: technology, historiography, and contextualizing Newness. Peter Lang, New York.

Parks, L. (2009): Digging into Google Earth: an analysis of "crisis in Darfur". In: Geoforum 40(4). S. 535-545.

Parks, L. \& N. Starosielski (2015): Introduction. In: dies. (Hrsg.): Signal Traffic. Critical Studies of Media Infrastructures. University of Illinois Press, Urbana. S. 1-27.

Passig, K. \& A. Scholz (2015): Schlamm und Brei und Bits: warum es die Digitalisierung nicht gibt. In: Merkur 69(798). S. 75-81.

Perkins, C. (2014): Plotting practices and politics: (im)mutable narratives in OpenStreetMap. In: Transactions of the Institute of British Geographers 39(2). S. 304-317.
Poorthuis, A. \& M. Zook (2014): Spaces of volunteered geographic information. In: Adams, P. \& J. Craine (Hrsg.): Ashgate research companion media geography. Routledge, London. S. 311-328.

Poster, M. (2006): Information please: culture and politics in the age of digital machines. Duke University Press, Durham, NC.

Rid, T. (2016): Maschinendämmerung. Eine kurze Geschichte der Kybernetik. Propyläen Verlag, Berlin.

Rifkin, J. (2011): Die dritte Industrielle Revolution. Die Zukunft der Wirtschaft nach dem Atomzeitalter. Campus, Frankfurt a. M.

Robertson, R. (1995): Glocalization: time-space and homogeneity-heterogeneity. In: Featherstone, M., S. Lash \& R. Robertson (Hrsg.): Global modernities. Sage, London. S. 25-44.

Seyfert, R. \& J. Roberge (2017) (Hrsg.): Algorithmuskulturen. Über die rechnerische Konstruktion der Wirklichkeit. Transcript, Bielefeld.

Soja, E. W. (2010): Seeking spatial justice. University of Minnesota Press, Minneapolis.

Starosielski, N. (2015a): The undersea network. Duke University Press, Durham.

Starosielski, N. (2015b): Fixed Flow. Undersea Cables as Media Infrastructure. In: Parks, L. \& N. Starosielski (Hrsg.): Signal Traffic. Critical Studies of Media Infrastructures. University of Illinois Press, Urbana. S. 53-70.

Stephens, M. (2013): Gender and the GeoWeb: divisions in the production of user-generated cartographic information. In: GeoJournal 78(6). S. 981-996. https://doi. org/10.1007/s10708-013-9492-z (zuletzt abgerufen am 29.01.2019).

Sterly, H. (2018): Regionalization revisited. Mediatization of translocal social practices and the spatial reconfiguration of life in rural-urban Bangladesh. In: Felgenhauer, T. \& K. Gäbler (Hrsg.): Geographies of Digital Culture. Routledge, London. S. 52-68.

Thrift, N. \& S. French (2002): The automatic production of space. In: Transactions of the Institute of British Geographers 27(3). S. 309-335.

Torrens, P. M. (2008): Wi-Fi geographies. In: Annals of the Association of American Geographers 98(1). S. 59-84.

Tranos, E. (2013): The geography of the internet: cities, regions, and internet infrastructure in Europe. Edward Elgar Publishing, Cheltenham.

Warf, B. (2018): Digital divides in the twenty-first century United States. In: Felgenhauer, T. \& K. Gäbler (Hrsg.): Geographies of Digital Culture. Routledge, London. S. 115-130.

Werlen, B. (2008): Sozialgeographie. Eine Einführung. Haupt, Bern. 